\title{
BMJ Open Interventions to improve early cancer diagnosis of symptomatic individuals: a scoping review
}

\author{
George N Okoli (D) , ${ }^{1}$ Otto L T Lam (1) , , Viraj K Reddy (1) , ${ }^{1}$ Leslie Copstein (1) , \\ Nicole Askin (D) , ${ }^{2}$ Anubha Prashad, ${ }^{3}$ Jennifer Stiff, ${ }^{3}$ Satya Rashi Khare, ${ }^{3}$ \\ Robyn Leonard, ${ }^{3}$ Wasifa Zarin, ${ }^{4}$ Andrea C Tricco (D) , $4,5,6$ \\ Ahmed M Abou-Setta (1) 1,7
}

To cite: Okoli GN, Lam OLT, Reddy VK, et al. Interventions to improve early cancer diagnosis of symptomatic individuals: a scoping review. BMJ Open 2021;11:e055488. doi:10.1136/ bmjopen-2021-055488

- Prepublication history and additional supplemental material for this paper are available online. To view these files, please visit the journal online (http://dx.doi.org/10.1136/ bmjopen-2021-055488)

Received 13 July 2021 Accepted 21 October 2021

Check for updates

(c) Author(s) (or their employer(s)) 2021. Re-use permitted under CC BY-NC. No commercial re-use. See rights and permissions. Published by BMJ.

For numbered affiliations see end of article.

Correspondence to

Dr George N Okoli;

george.okoli@umanitoba.ca

\section{ABSTRACT}

Objectives To summarise the current evidence regarding interventions for accurate and timely cancer diagnosis among symptomatic individuals.

Design A scoping review following the Joanna Briggs Institute's methodological framework for the conduct of scoping reviews and reported in accordance with the Preferred Reporting Items for Systematic Reviews and Meta-Analyses extension for Scoping Reviews checklist. Data sources MEDLINE (Ovid), CINAHL (EBSCOhost) and PsycINF0 (Ovid) bibliographic databases, and websites of relevant organisations. Published and unpublished literature (grey literature) of any study type in the English language were searched for from January 2017 to January 2021.

Eligibility and criteria Study participants were individuals of any age presenting at clinics with symptoms indicative of cancer. Interventions included practice guidelines, care pathways or other initiatives focused on achieving predefined benchmarks or targets for wait times, streamlined or rapid cancer diagnostic services, multidisciplinary teams and patient navigation strategies. Outcomes included accuracy and timeliness of cancer diagnosis.

Data extraction and synthesis We summarised findings graphically and descriptively.

Results From 21298 retrieved citations, 88 unique published articles and 16 unique unpublished documents (on 18 study reports), met the eligibility for inclusion. About half of the published literature and $83 \%$ of the unpublished literature were from the UK. Most of the studies were on interventions in patients with lung cancer. Rapid referral pathways and technology for supporting and streamlining the cancer diagnosis process were the most studied interventions. Interventions were mostly complex and organisation-specific. Common themes among the studies that concluded intervention was effective were multidisciplinary collaboration and the use of a nurse navigator.

Conclusions Multidisciplinary cooperation and involvement of a nurse navigator may be unique features to consider when designing, delivering and evaluating interventions focused on improving accurate and timely cancer diagnosis among symptomatic individuals. Future research should examine the effectiveness of the interventions identified through this review.
Strengths and limitations of this study

- A knowledge synthesis librarian developed the search strategy for this review and this was peerreviewed by an independent knowledge synthesis librarian using the Peer Review of Electronic Search Strategies checklist.

- The literature search was limited to evidence from the last 4 years and only evidence from Englishlanguage publications and organisational websites.

- This review did not summarise the effectiveness of interventions across cancer patient types and regions.

- We adhered to known guidelines and standards in the conduct and reporting of the review.

- In line with the Joanna Briggs Institute's guidance for the conduct of scoping reviews, we did not attempt to evaluate the quality of the included studies or provide an assessment of the quality of the evidence.

\section{INTRODUCTION}

Cancer is the second leading cause of death globally, with about one in six deaths attributable to the disease. ${ }^{1}$ It was estimated in 2020 that over 19 million new cases and about 10 million deaths were attributable to cancer globally. ${ }^{2}$ This rate is estimated to be over 28 million new cases by $2040 .^{2}$ High Human Development Index countries such as Canada will likely experience the greatest increase in incidence in absolute cancer burden, with an estimated over 4 million new cases more in 2040 compared with $2020 .^{2}$ This is mostly due to the growth and ageing of the population and increasing prevalence of cancer risk factors. ${ }^{2}$ Estimates from Canada alone suggest that every day 617 people in Canada will be diagnosed with cancer, with about 228 also dying from the disease. ${ }^{3}$

Although cancer can occur at any age, the risk of the disease increases with age. ${ }^{4}$ Globally, cancer incidence rates vary, mostly because of 
differences in risk factors and early detection practices. Likewise, cancer death rates vary, partly because of differences in availability and effectiveness of cancer control strategies, such as early diagnosis and access to timely and effective treatment. ${ }^{2}$ With timely diagnosis and treatment initiation, significant improvements can be made in the lives of patients with cancer. Moreover, many cancers have higher curative and survival rates if diagnosed early. This means that the cancer burden could be reduced substantially through early detection and management of patients who present with symptoms. ${ }^{5}$

When not diagnosed following early symptomatic presentation, cancer diagnosis often occurs at more advanced stages of the disease, when treatment may be less effective and cancer prognosis will be poor. Early cancer diagnosis of symptomatic individuals entails carefully planned, well-integrated, culturally safe and equitable clinical evaluation and diagnostic services. ${ }^{5}$ These services should be designed to reduce delays in and barriers to diagnosis to allow detection at earlier stages of the disease and commence treatment in a timely manner.

Various service-focused interventions to improve early cancer diagnosis of symptomatic individuals have been implemented in various jurisdictions with varying levels of success. Knowledge of the available interventions, strategies used to implement them, and how successful they might have been is necessary to inform the development, implementation and evaluation of effective early cancer diagnosis initiatives.

\section{METHODS}

This report is a summary of the study commissioned by the Canadian Partnership Against Cancer (the Partnership). The Partnership contributed to specifying the study objectives and questions, and in summarising the evidence.

We undertook a scoping review following the Joanna Briggs Institute's (JBI's) guidance for the conduct of scoping reviews. ${ }^{6}$ This framework includes defining and aligning the objective(s) and question(s) for the review, developing and aligning the inclusion criteria with the review objective(s) and question(s), and describing the planned approach to evidence searching. It also includes selecting, extracting and charting of evidence; summarising the evidence in relation to the objectives and questions; and consultation of information scientists, librarians and/or experts throughout the process. Online supplemental appendix 1 is the work plan approved by the Partnership for the scoping review.

We summarised the current evidence regarding interventions focused on improving accurate and timely cancer diagnosis among symptomatic individuals, including practice guidelines, care pathways or targets for wait times, streamlined or rapid diagnostic services, multidisciplinary teams, and patient navigation strategies. We also summarised innovative interventions (eg, those with a technological component) and approaches to seamless (minimally disruptive) care of symptomatic individuals and identified performance metrics that can be used to measure improvements in the prediagnosis phase. Additionally, we summarised the key points of the patient trajectory from initial symptom presentation to cancer diagnosis.

We report our findings in accordance with the Preferred Reporting Items for Systematic Reviews and Meta-Analyses extension for Scoping Reviews (PRISMA-ScR) checklist. ${ }^{7}$

\section{Search strategy}

A knowledge synthesis librarian (NA) designed a search strategy for MEDLINE (Ovid). This search strategy was peer-reviewed independently by another knowledge synthesis librarian using the Peer Review of Electronic Search Strategies (PRESS) checklist. ${ }^{8}$ The revised search strategy was then adapted for Cumulative Index to Nursing and Allied Health Literature (CINAHL) (EBSCOhost) and PsycINFO (Ovid) bibliographic databases. The search strategy for each of the databases is presented in online supplemental appendices 2-4. In addition to searching bibliographic databases, we searched websites of relevant organisations and professional bodies (online supplemental appendix 5) and hand-searched reference lists of potentially relevant publications.

\section{Study selection criteria and data extraction}

We sought to summarise practice guidelines, care pathways and initiatives such as benchmarks/targets for wait times, streamlined or rapid diagnostic services, multidisciplinary teams and patient navigation strategies that have been found to enhance accurate and timely cancer diagnosis in symptomatic individuals. We also sought to summarise the leading interventions to seamless care in the cancer prediagnosis phase, performance metrics that can be used to measure the suspicion to diagnosis phase and how these metrics have been used. Further, we sought for specific considerations for underserviced populations in studies, including considerations for Indigenous, rural and remote populations.

Published (peer-reviewed) and unpublished (grey literature) articles in the English language from January 2017 to January 2021 were included. The decision to include articles from 2017 was because the Partnership had previously summarised prior evidence, not included in this current report. ${ }^{9}$ Study participants were individuals of any age presenting in any clinical settings with symptoms. Interventions included practice guidelines, care pathways or other initiatives focused on achieving predefined benchmarks or targets for wait times, streamlined or rapid diagnostic services, multidisciplinary teams and patient navigation strategies. Outcomes included accuracy and timeliness of cancer diagnosis.

All retrieved citations from the literature search were imported and managed in EndNote (V.X9). One reviewer (GNO or OLTL or VKR or LC) screened each citation for eligibility. Two reviewers (GNO, OLTL, VKR, and LC in pairs) independently screened the full texts 
of relevant citations and reviewed the reference list of the included full-text articles for potentially relevant citations. Disagreements between the reviewers were resolved through discussion or involvement of a third reviewer (AMA-S). The number of screened citations and both the number and reason for exclusion of full-text articles were documented. One reviewer (GNO or OLTL or VKR or LC) performed data extraction and charting, and another reviewer (GNO or OLTL or VKR or LC) independently checked the extracted and charted data for errors. Disagreements between the reviewers were resolved through discussion or involvement of a third reviewer (AMA-S).

\section{Data synthesis and analysis}

Characteristics of the included published articles are presented in a tabular form and descriptive analysis is reported graphically and descriptively. Characteristics of the included unpublished articles are reported descriptively only. Relevant findings from the review of both published and unpublished articles are summarised separately and descriptively, by review question, focusing on the interventions related to each question. Interventions are grouped as centralised or coordinated diagnostic service; interventions to enhance diagnostic services; multidisciplinary team; patient navigation; rapid referral pathway; remote or rural populations-focused; standardised care pathway; support for primary care providers (PCP); target or benchmark; and technology to support the diagnostic process. These interventions are defined in online supplemental appendix 6 . We determined the effectiveness of an intervention based on study findings and conclusions reported by the primary study's authors with respect to intervention effect. As such, effective interventions were those interventions that were found to have had a statistically significant positive effect on an author-determined outcome for effectiveness evaluation. It is important to note that the authors of this scoping review did not assess risk of bias nor rate the quality of evidence and thus definitive conclusions on effectiveness cannot be drawn.

\section{Patient and public involvement}

There was no active engagement of patients and/or members of the public.

\section{RESULTS}

Out of a total of 21298 retrieved citations, 88 unique published articles ${ }^{10-97}$ and 16 unique unpublished (grey literature representing 18 different reports) ${ }^{98-113}$ met the inclusion criteria. The article selection process is detailed below (figure 1). Fifty-seven of the published articles were from Europe, 14 articles from North America, 9 articles from Oceania, 3 articles each from Africa and Asia and 1 article each from the Middle East and South America. Almost half of these articles $(n=40)$ were from the UK alone. A geographic map of published articles is shown in figure 2.

Of the 18 unpublished reports (16 articles), $83 \%$ were from the UK, $11 \%$ from Canada and $6 \%$ from the USA. Forty per cent $(n=35)$ of the published articles were for case-control studies, 29\% $(\mathrm{n}=26)$ for cross-sectional studies, 22\% (n=19) for before-and-after studies, $7 \%$ $(n=6)$ for randomised controlled studies and $1 \%(n=1)$ each for guideline development and mixed methods studies. In terms of the unpublished articles, $89 \%(n=16)$ were before-and-after studies and the rest $(n=2)$ were cross-sectional studies. Figure 3 shows the distribution of the cancer types reported by the published articles; approximately $30 \%(\mathrm{n}=26)$ reported on multiple cancer types, while the rest reported on specific cancer types, of which lung cancer was the most frequent (about 23\% of the publications $(n=20))$. Of the unpublished articles, half reported on lung cancer, $28 \%$ on multiple cancer types, $11 \%$ on breast cancer and $5.5 \%$ each on brain and gastrointestinal cancers.

Figure 4 shows the distribution of intervention types across the published articles. Nearly $20 \%$ of the published articles were on rapid referral pathway interventions while less than $1 \%$ each were on multidisciplinary team, patient navigation and remote/rural-focused interventions. Of the unpublished articles, half reported on rapid referral pathway interventions, $11 \%$ each reported on standardised care pathway, target/ benchmark for wait times and technology to support the diagnosis process, and $5.5 \%$ each reported on centralised or coordinated diagnostic service and interventions to enhance diagnostic services. Most of the published articles (94\%; $\mathrm{n}=83$ ) reported a performance metric used to measure an improvement in the suspicion to diagnosis phase of cancer.

Eighty-three per cent $(n=73)$ of the articles reported either a practice guideline, care pathway or an initiative such as benchmark/target for wait times, streamlined or rapid diagnostic service, multidisciplinary team development and a patient navigation strategy to enhance accurate and timely cancer diagnosis. Thirty-one per cent $(n=27)$ of the articles reported (not explicitly) on a key point of care as patients navigate the health system, from initial suspicion to diagnosis of cancer. Twenty-nine per cent $(n=25)$ of the articles reported on a leading innovative intervention or approach to seamless care in the precancer diagnosis phase, while $4.5 \%$ $(n=4)$ of the articles reported on some form of consideration for underserved populations. Some of the articles reported on two or more of the above. Details of relevant characteristics of the published articles are presented in table 1 (those reporting effective interventions) and online supplemental appendix 7 (those reporting ineffective interventions) and online supplemental appendix 8 (those focused on remote/and rural populations). 

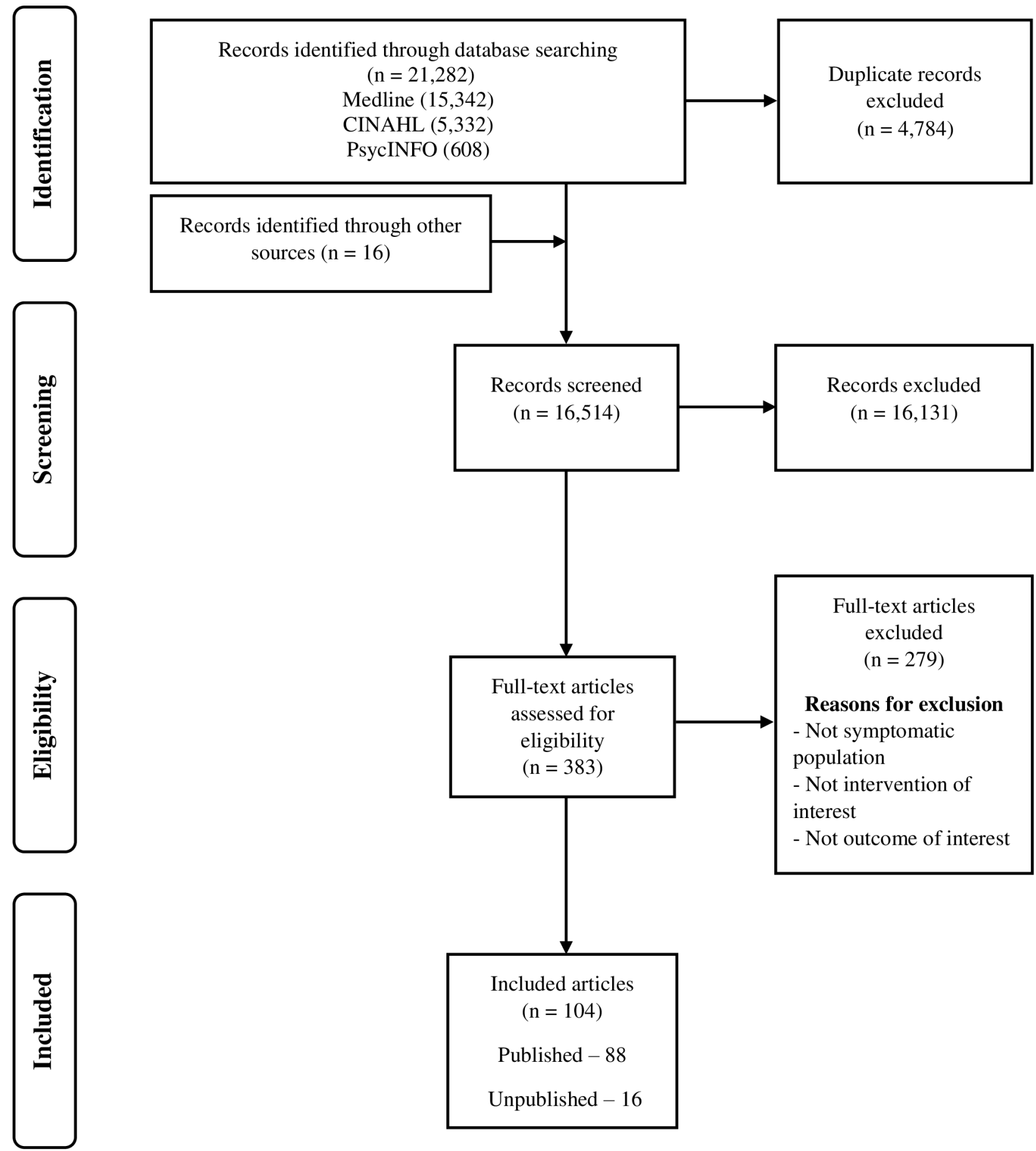

Figure 1 Modified Preferred Reporting Items for Systematic Reviews and Meta-Analyses flow chart.

Initiatives to enhance accurate and timely cancer diagnosis This review identified various initiatives to enhance accurate and timely cancer diagnosis. These were often designed, developed and implemented often with the involvement of PCP (physicians and nurses), but not patients. These initiatives are grouped into related interventions and the evidence regarding each intervention is discussed below.

\section{Centralised or coordinated diagnostic services}

Nine published articles on centralised or coordinated diagnostic services for adult lung cancer $(n=5)$ and breast cancer $(\mathrm{n}=4)$ patients were identified. ${ }^{202332334454-5693}$ Five were from Canada, ${ }^{23} 33445455$ and there was one each from Denmark ${ }^{20}$ New Zealand ${ }^{93}$ South Africa ${ }^{56}$ and the UK. ${ }^{32}$ The focus and metrics for assessment of the effectiveness of these diagnostic services varied, but all were found to be effective. These include the rapid access to pulmonary investigation and diagnosis programme in Wythenshawe Hospital, Manchester, UK with expedited (next working day) CT and reporting in suspected lung cancer cases, ${ }^{32}$ and the Thoracic Triage Panel in a tertiary care centre in St. John's, Newfoundland, Canada, a multidisciplinary 


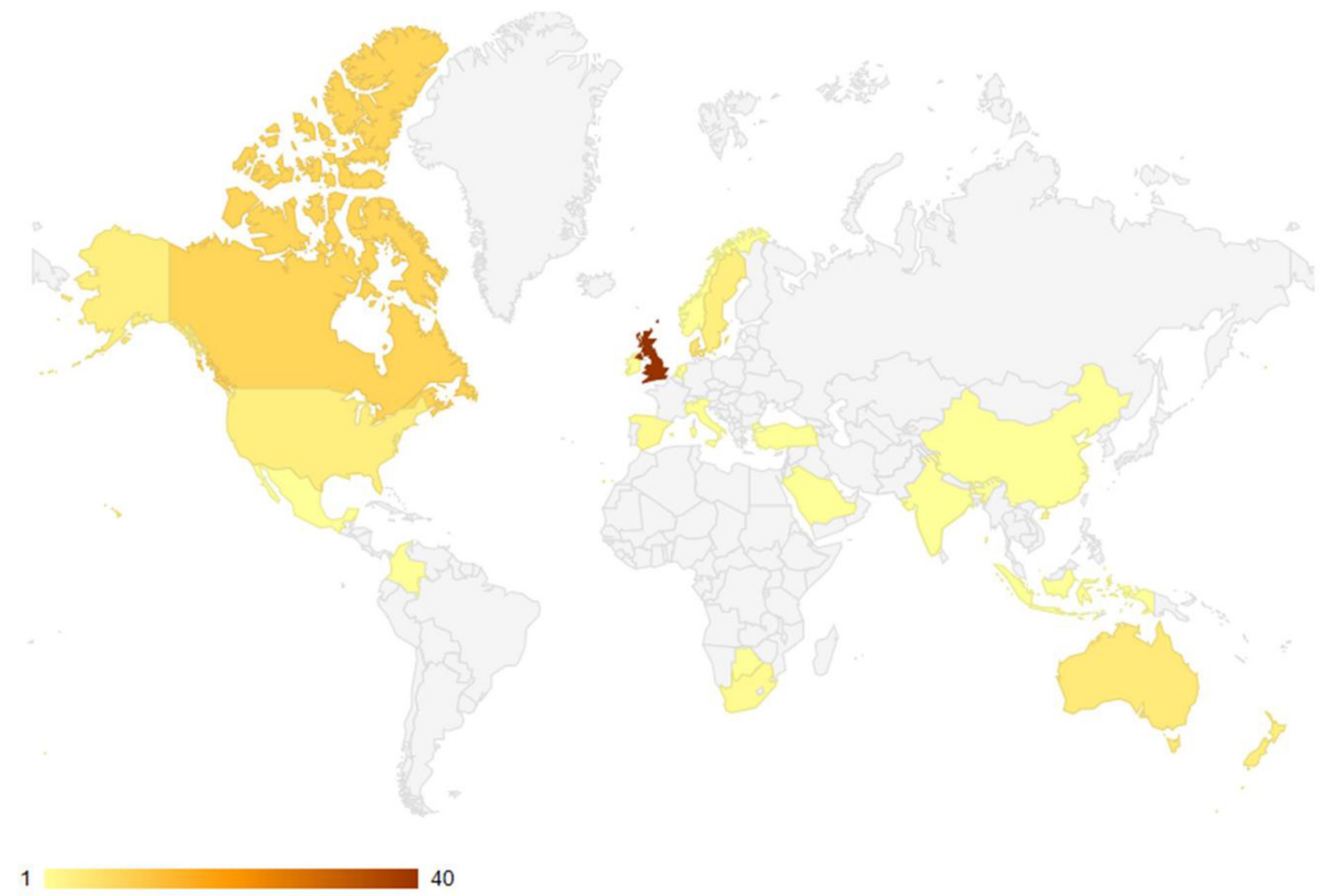

Figure 2 Geographical mapping of the included published articles.

centralised referral programme, whose key components include a nurse navigator who coordinates patient care and act as the contact person for patients and clinicians involved in the programme, weekly multidisciplinary (thoracic specialists) meetings and regular communications with the primary care provider. ${ }^{23}$ The diagnostic services also include the rapid investigation clinic in a tertiary health centre in Montreal, Canada established to coordinate and accelerate the workup of patients with suspected lung cancer, ${ }^{33}$ the improved respiratory fast track clinic in Northland district of New Zealand that comprises reserved slots for $\mathrm{CT}$ for those referred with a suspicion of lung cancer, bronchoscopy slots and CT-guided biopsy, ${ }^{93}$ and the Danish lung cancer package at the Center for Lung Cancer, Odense University Hospital, Odense, Denmark, a fast-track diagnostic pathway in the hospital setting. ${ }^{20}$ Further, there was the rapid access breast clinic in British Columbia, Canada that provides close collaboration between clinicians and radiologists, facilitated by clinical pathways and nurse navigation, ${ }^{54}{ }^{55}$ the diagnostic assessment units in Ontario, Canada, focusing on diagnosis at a dedicated breast assessment unit, ${ }^{44}$ and the breast clinic at a tertiary hospital in Western Cape Province of South Africa, an open-access one-stop diagnostic breast clinic where women may present with a letter from a primary level provider (nurse practitioner or doctor) and receive the same day clinical and cytological evaluation with referral to the combined breast clinic if the breast cytology is positive for malignancy. ${ }^{56}$
In addition to the above, one unpublished article was identified. ${ }^{113}$ This was for the Breast ACCESS Project in Ohio, USA, which scheduled patients for a surgical consult within 2 days and a biopsy within 5 days after the surgical consult, with the aim of reducing wait times between abnormal diagnostic mammogram findings to biopsy from 26 to 7 days (7-day ACCESS goal).

\section{Interventions to enhance diagnostic services}

Twelve published articles on interventions to enhance diagnostic services were identified. ${ }^{10} 1724525364757778808394$ These articles were focused on varied cancer types; four on multiple cancers, two on lung cancer, two on skin cancer and one each on breast, gastrointestinal, haematological and prostate cancers. Four articles were from the UK, ${ }^{1752} 5378$ two articles each from Canada ${ }^{2464}$ and Sweden, ${ }^{10}{ }^{80}$ and one article each from Botswana, ${ }^{94}$ Columbia, ${ }^{75}$ Indonesia ${ }^{77}$ and the USA. ${ }^{83}$ The focus and metrics for assessment of the effectiveness of the interventions varied across the publications, and while most were effective, one intervention for lung cancer and one intervention for skin cancer in the $\mathrm{UK}^{53}$ and Sweden, ${ }^{10}$ respectively, were ineffective. The effective interventions were reducing diagnosis through emergency presentation by improving general practice referral in England, UK, ${ }^{52}$ the guided personal quality of life (QoL) feedback intervention during the Cancer Research UK's North West regional summer roadshow in Manchester, UK, aimed at offering guided feedback about personal QoL to adults with 


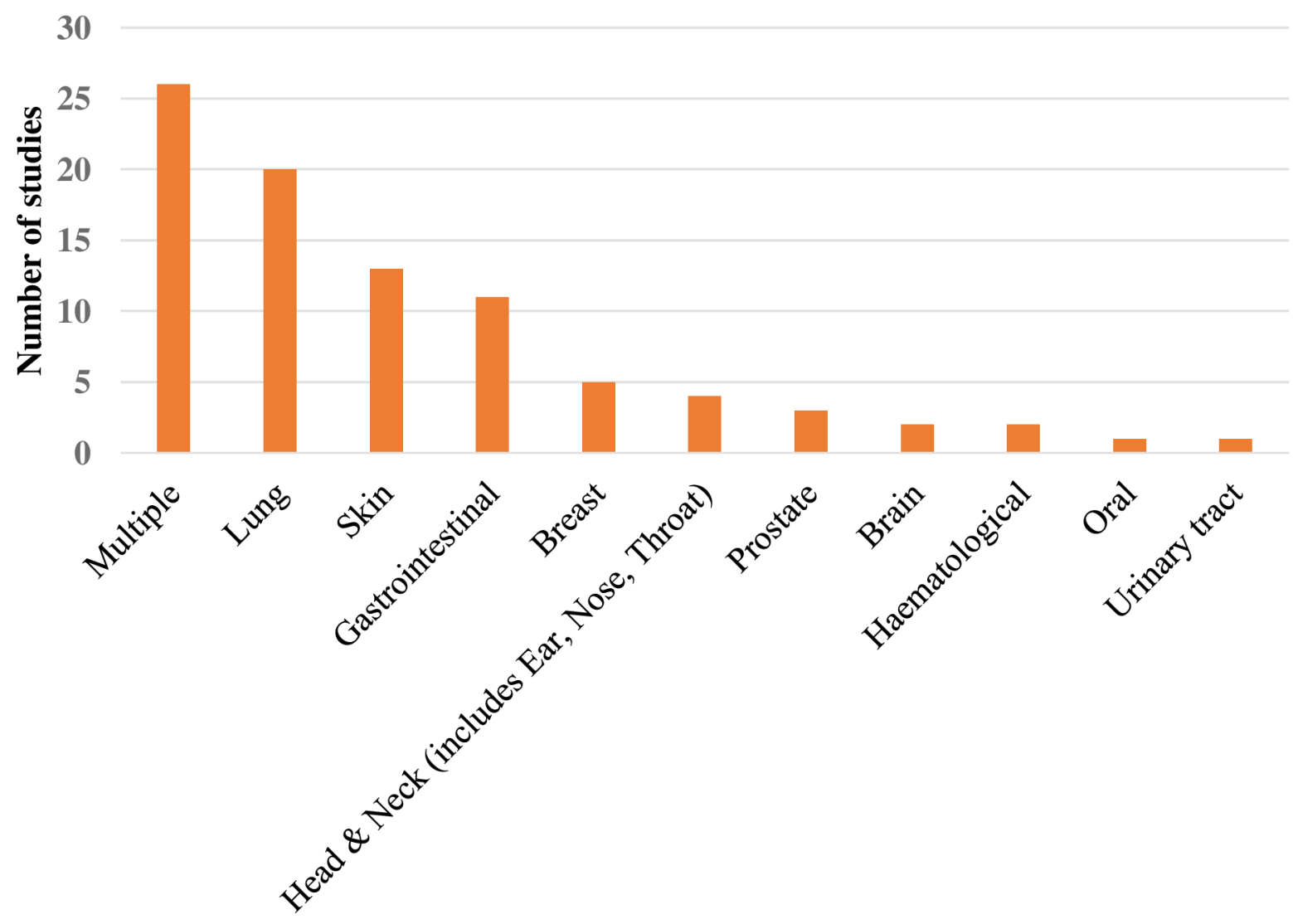

Cancer type

Figure 3 Summary of cancer types reported by the included published articles.

potential cancer symptoms, living in deprived communities to promote help seeking in primary care among the communities, ${ }^{78}$ the mandatory primary care access to faecal immunochemical testing in Nottingham, UK, integrated with the 2-week wait pathway, aimed at improving gastrointestinal cancer diagnosis rather than relying on age and symptoms alone,${ }^{17}$ the Stronach Regional Cancer Centre lung diagnostic assessment programme at Southlake Regional Health Centre, Ontario, Canada, aimed at using learnings from a Lean improvement event to provide coordinated, expedited care for all patients undergoing a possible lung cancer diagnosis and to achieve/improve on the provincial wait time target from consultation to diagnosis for patients with lung cancer, ${ }^{24}$ the nurse practitioner-led lymphoma rapid diagnosis clinic in a tertiary care cancer centre (Princess Margaret Cancer Centre, part of University Health Network) in Ontario, Canada, aimed at reducing wait times for a definitive diagnosis of lymphoma, ${ }^{64}$ the expedited one-stop prostate cancer diagnosis using advanced imaging and biopsy techniques in a health institution (name not reported) in the USA, aimed at expediting prostate cancer diagnosis. ${ }^{83}$ There was also the Swedish Diagnostic Center at the Central Hospital of Kristianstad, Sweden, introduced as a separate outpatient unit within the Department of Internal Medicine to expedite diagnostics, ${ }^{80}$ the Partners for Cancer
Care and Prevention action plan in Cali, Columbia, aimed at improving access to a coordinated programme of screening and early diagnosis of breast and cervical cancers in three healthcare centres that serve subsidised populations, ${ }^{75}$ the dermatology-led quality improvement initiatives in Gaborone, Botswana, aimed at improving multispecialty care coordination, ${ }^{94}$ and the culturally sensitive, narrative self-help intervention named PERANTARA (PEngantar peRAwataN kesehaTAn payudaRA (translated as introduction to breast health treatment)) across four hospitals in Bandung, West Java, Indonesia, aimed at reducing time to diagnosis in women with breast cancer symptoms. ${ }^{77}$ In addition to the above, one unpublished article on the Accelerate, Coordinate, Evaluate programme in the UK was identified. ${ }^{100}$ This programme was an early cancer diagnosis initiative and focused on testing innovations that either identify individuals at high risk of cancer earlier or streamline diagnostic pathways.

The ineffective interventions were the standardised care diagnostic pathway at the Department of Clinical Pathology, Akademiska University Hospital in Uppsala, Sweden (introduced by the Swedish health authorities to eliminate unwanted delay in the diagnostics of melanoma) ${ }^{10}$ and the 4 -week national lung cancer symptom awareness campaign in Wales, UK, aimed at increasing urgent suspected cancer referrals and clinical outcomes. ${ }^{53}$ 


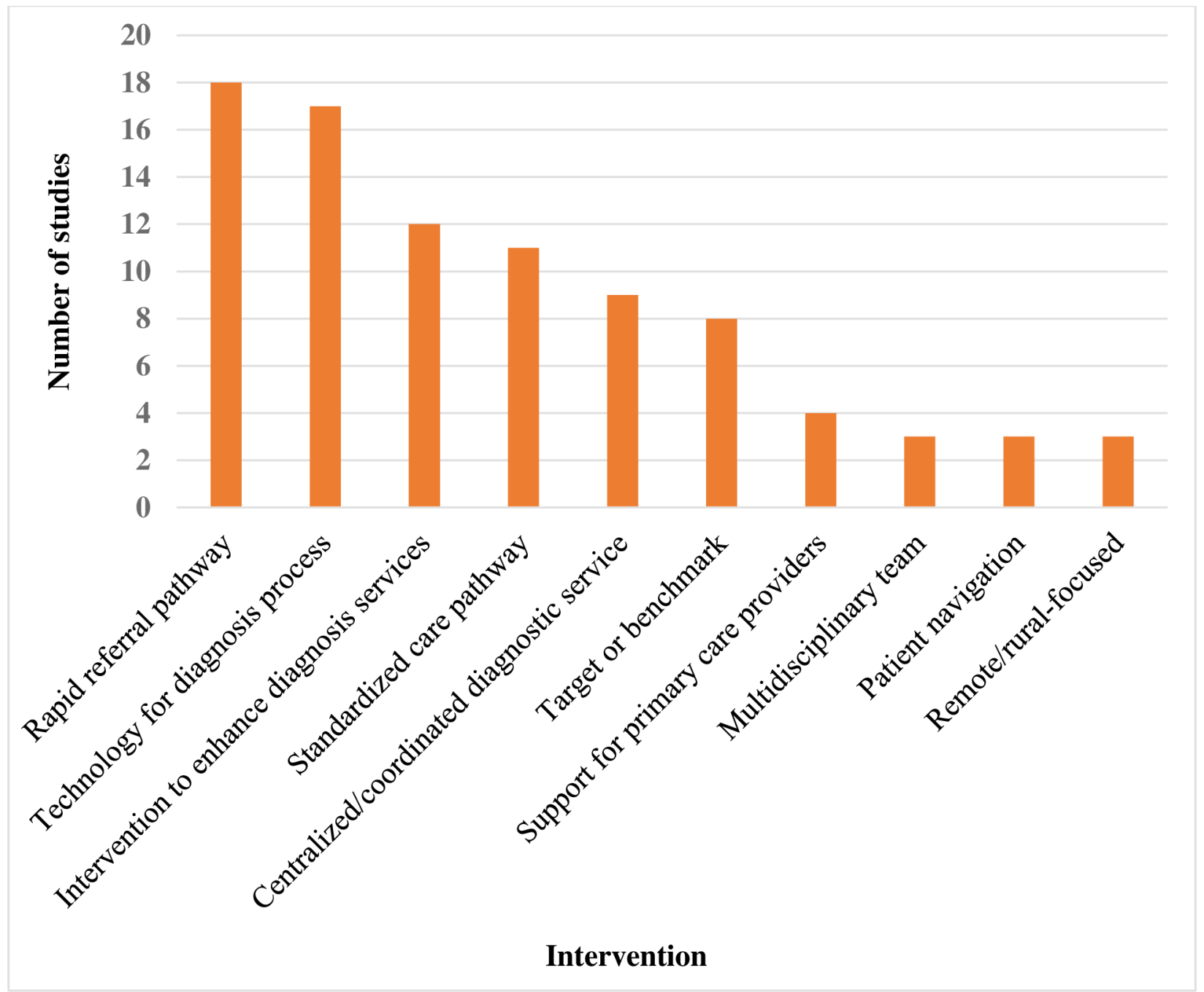

Figure 4 Summary of intervention types reported by the included published articles.

\section{Multidisciplinary team}

Three multidisciplinary team lung cancer approaches were identified from published articles: from the USA ${ }^{6885}$ and Australia. ${ }^{50}$ The focus and metrics for assessment of the effectiveness of the approaches varied across the publications. One approach from the USA was found to be effective ${ }^{68}$ whereas the others were found to be ineffective. The effective approach was the lung cancer strategist programme, a thoracic surgeon-guided, multidisciplinary (disciplines not reported) care programme in hospitals in Massachusetts, USA, aimed at improving timeliness of lung cancer diagnosis and treatment. ${ }^{68}$ The ineffective approaches were the pre-diagnosis multidisciplinary tumour board (physicians from radiology, medical and radiation oncology, and pulmonary medicine) discussions in a clinic in Cleveland, USA aimed at improving the timeliness of diagnostic evaluation in lung cancer, ${ }^{85}$ and the Victorian lung cancer service redesign project in Victoria, Australia, which involved multidisciplinary (patients, governance, administration, clinicians and health information services) evaluation aimed at quality improvement collaborative on timeliness and management in lung cancer. ${ }^{50}$ In addition, nine unpublished articles from the UK were identified. ${ }^{99}$ 101-103 106108109112 These included four articles regarding a 'straight to CT access' pathway, on community pharmacy direct referral to lung cancer pathway, rapid colorectal diagnostic pathway, and optometrist direct referral to neuroscience pathway. All but the chest X-ray pathway ${ }^{109}$ were found to be effective.

\section{Standardised care pathways}

Eleven published articles on standardised care pathways were identified. ${ }^{11} 12263539414959637071$ These articles were focused on varied cancer types (four each for multiple cancers, and one each for ear-nose-throat, urinary tract and gastrointestinal cancers). Three articles were from Denmark, ${ }^{26} 3941$ two from the $\mathrm{UK}^{35}{ }^{70}$ and one each from Canada, ${ }^{59}$ Norway, ${ }^{49}$ Sweden, ${ }^{63}$ Spain ${ }^{12}$ and Saudi Arabia. ${ }^{11}$ The publications were on adult patient populations with one also involving paediatric patients. The focus and metrics for assessment of the effectiveness of the pathways varied across the publications. The main effective pathways were the national diagnostic cancer pathway in Norway, with recommended maximum limits for time spent in the diagnostic process as well 


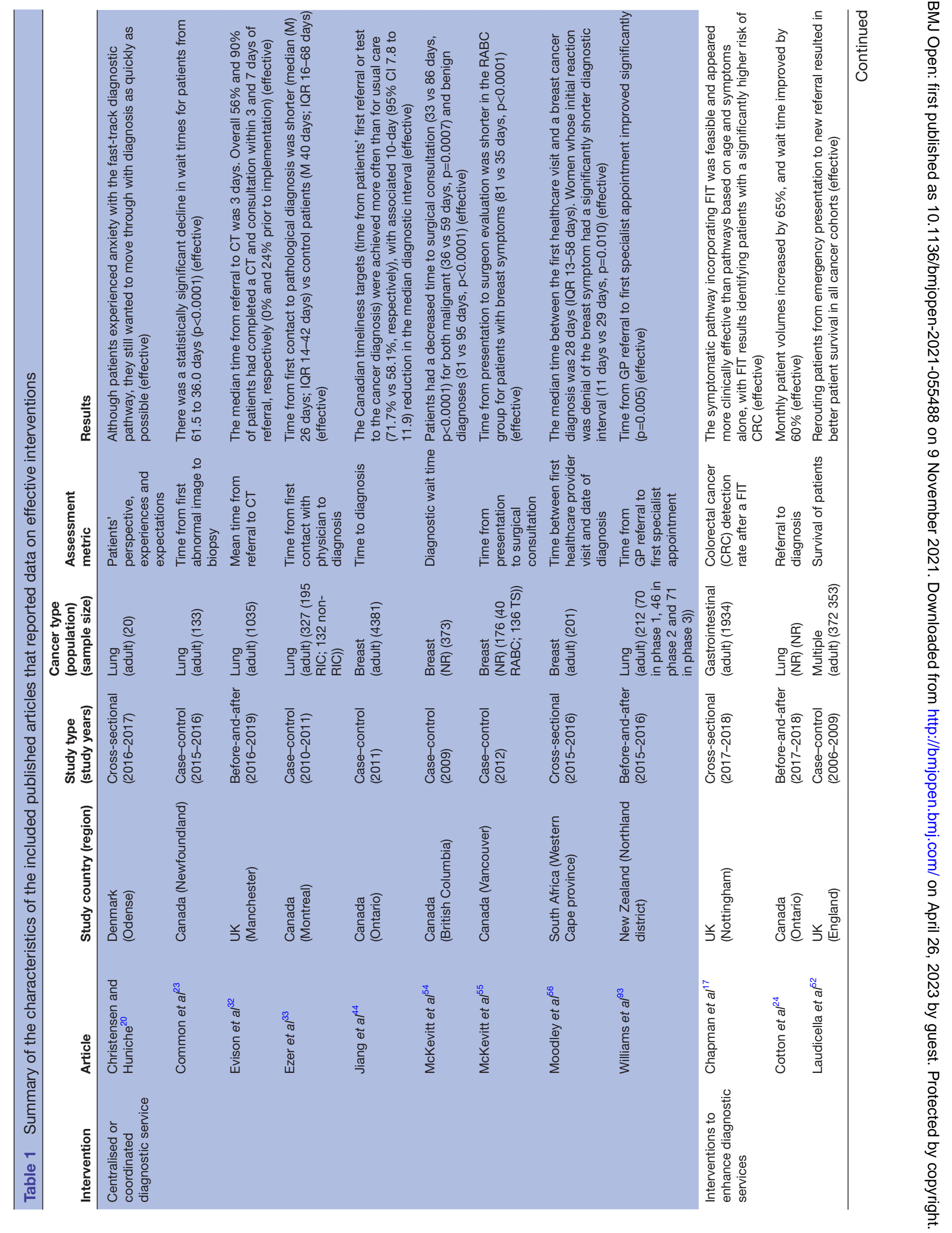




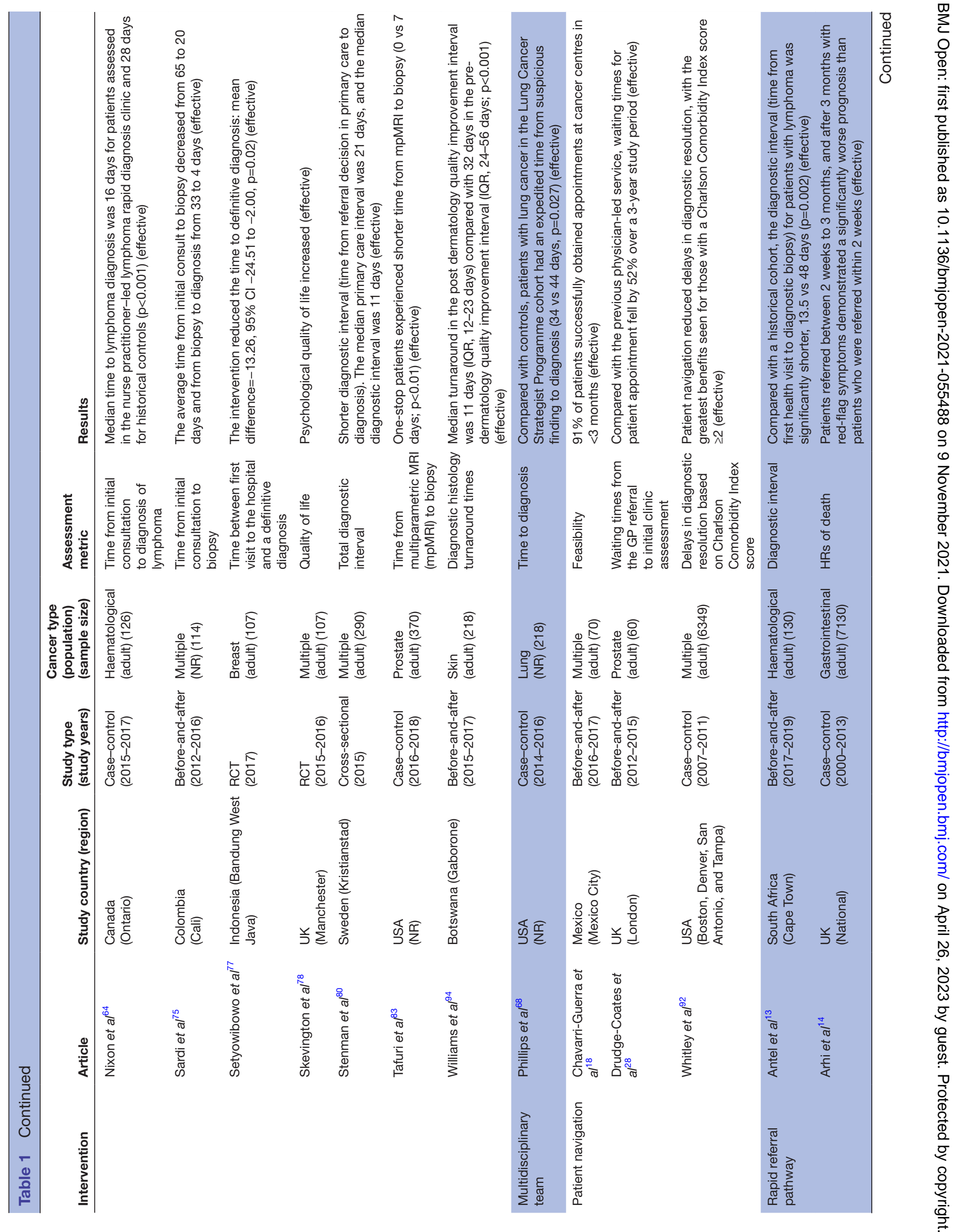




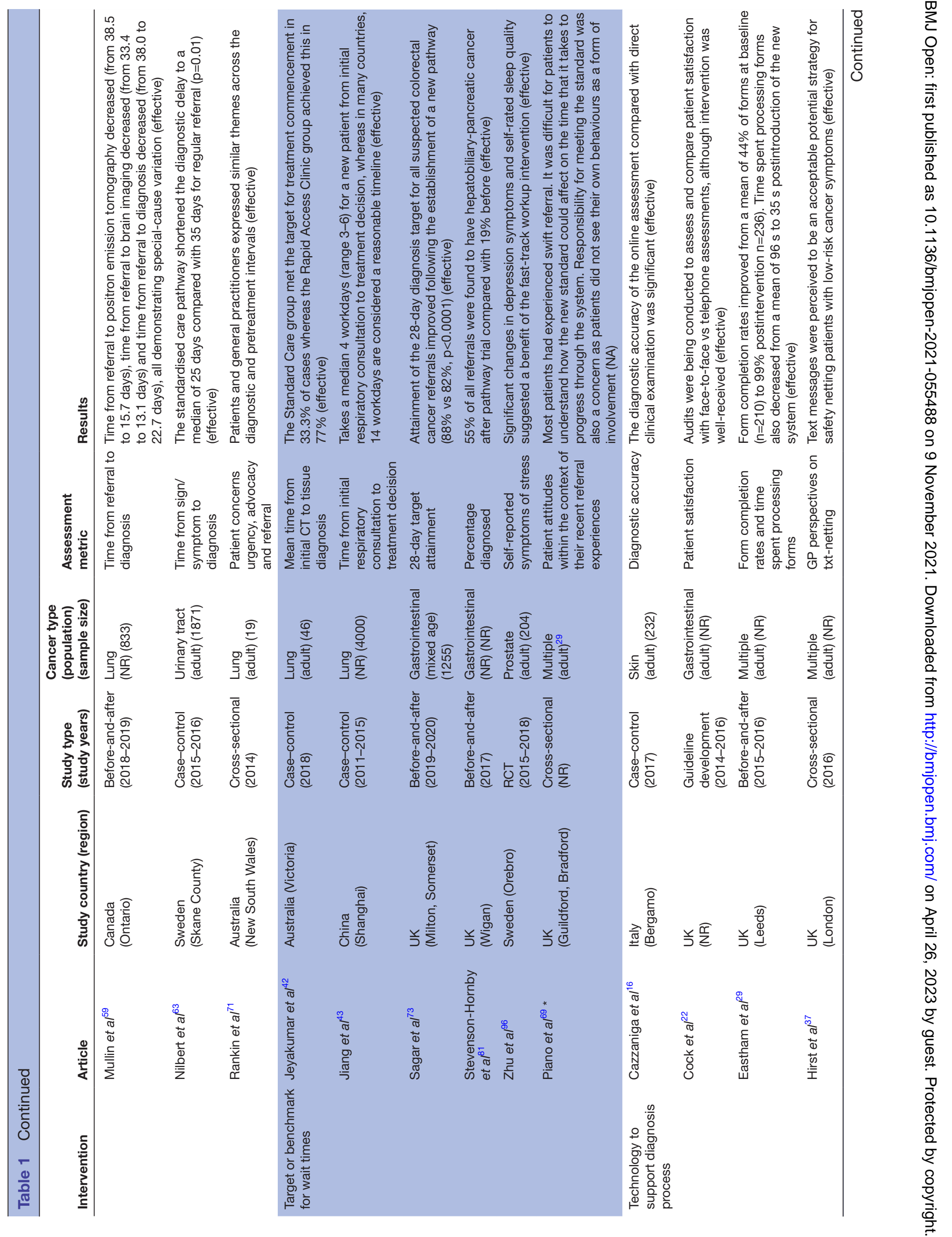




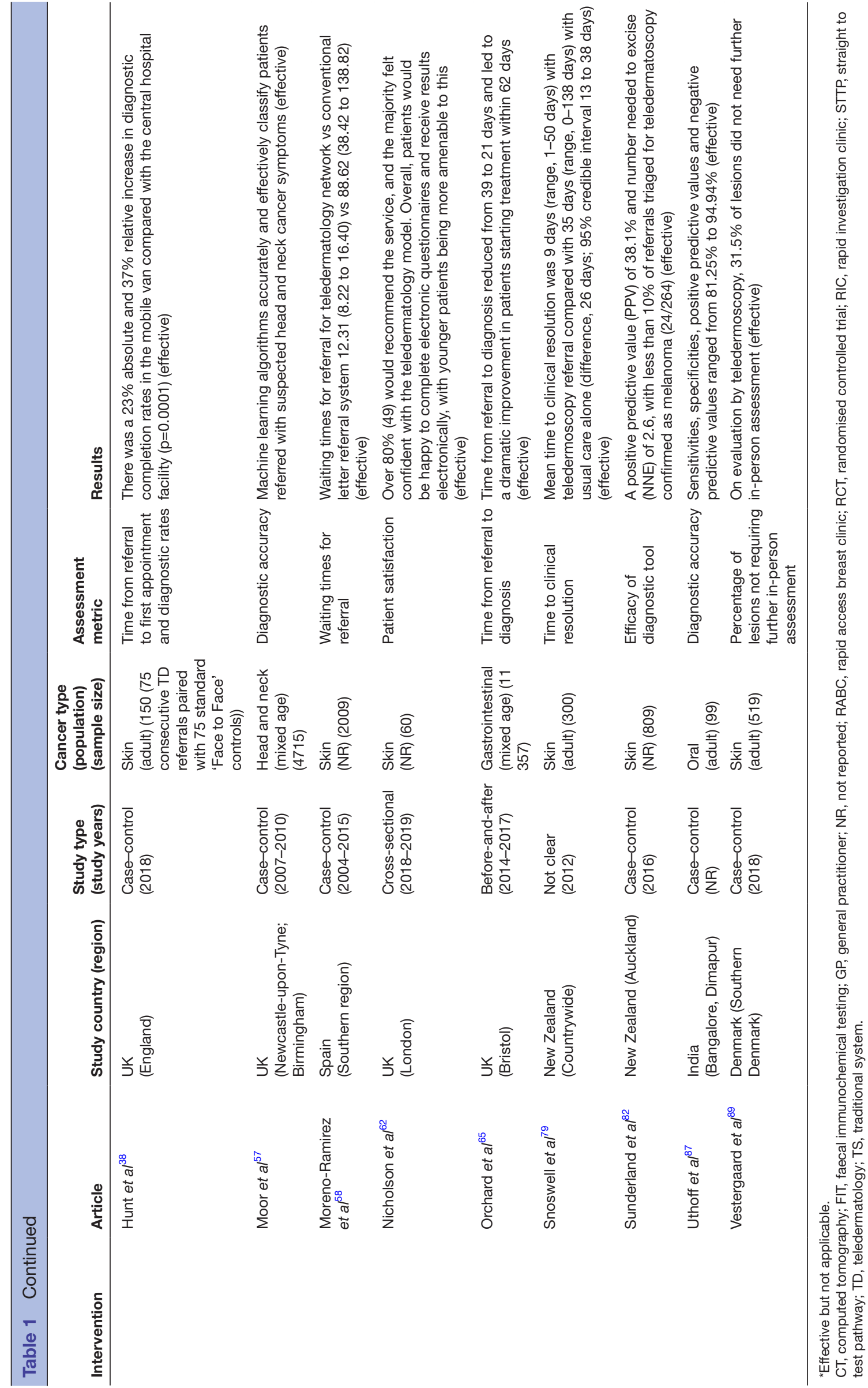


as mandatory reporting of the actual time intervals for all patients with suspected lung cancer, ${ }^{49}$ and the standardised triage process in the Southeastern Ontario, Canada, which entailed a two times-weekly nurse-physician triage, preordered staging tests and scheduling according to urgency, redirection and recommendations for inappropriate referrals, and new small nodule clinic. ${ }^{59}$ Other main effective pathways were the standardised diagnostic pathway for suspected urothelial cancer initiated by primary healthcare providers and specialists in Skane County, Sweden and comprises CT urography, urinary cytology and cystoscopy ${ }^{63}$ the early colonoscopy track (within 30 days from referral) in a tertiary referral hospital in Tenerife, Spain, ${ }^{12}$ and the fast-track cancer care pathway in Denmark (national), with maximum acceptable time thresholds from referral to diagnosis and treatment. ${ }^{39}$ In addition, two unpublished articles from Canada $^{111}$ and the $\mathrm{UK}^{98}$ focusing on breast and lung cancers, respectively, were identified. These were the Alberta Health Services Diagnostic Assessment Pathway and the Somerset Integrated Lung Cancer Pathway. While the Canadian pathway was found to be effective, the pathway from the UK was not effective.

\section{Support for PCP}

There were four publications on support for PCP, all from the UK. ${ }^{27} 314897$ Two were focused on multiple cancer types, and one each focused on gastrointestinal and brain cancers. The publications were on adult patient populations with one being also involving paediatric patients. The focus and metrics for assessment of the effectiveness of the support packages (all educational and informational) varied across the publications. None of the support packages was found to be effective, with the identified common theme being a lack of awareness of referral guidelines and associated knowledge by general practitioners (GPs). These ineffective support packages were the use of the Kernick and NICE guidelines as evidencebased support to assist primary care physicians in identifying patients most at risk of having a brain tumour, but also on the fastest route to achieve diagnosis (eg, direct access imaging vs urgent secondary care referral) in Scotland, the UK, ${ }^{97}$ the use of the national cancer waiting times monitoring dataset for system performance assessment by primary care physicians in England, the UK, ${ }^{27}$ and the use of safety netting by primary care physicians in Oxfordshire, UK to ensure that patients are monitored until their symptoms or signs are explained, and to guard against delays in diagnosis. ${ }^{31}$

\section{Target or benchmark for wait times}

There were eight published articles related to targets or benchmarks for wait times. ${ }^{15} 42436973818896$ Three of these articles were from the UK, ${ }^{69} 7381$ two articles from Australia ${ }^{428}$ and one article each from China, ${ }^{43}$ Sweden $^{96}$ and New Zealand. ${ }^{15}$ These publications were focused on varied cancer types (two each for multiple, lung and gastrointestinal cancers, and one each for prostate and skin cancers), and were on adult patient populations, with one publication involving paediatric patients. The focus and metrics for assessment of the effectiveness of the target or benchmarks varied across the publications, and all but two targets/benchmarks ${ }^{1588}$ were found to be effective. The effective targets or benchmarks were the 28-day faster diagnosis standard in the National Health Service England, UK, defined as the time within which the patient is informed whether they do or do not have cancer, ${ }^{73}$ the fast-track diagnostic workup for men with suspected prostate cancer at the Urology Department at Orebro University Hospital in Sweden, which entailed targeting the shortest possible waiting-time for a diagnostic workup process, ${ }^{96}$ and the optimal timeframes for referral and diagnosis of lung lesion at Latrobe Regional Hospital in Victoria, Australia established by the National Cancer Expert Reference Group as part of the optimal care pathway for people with lung cancer. ${ }^{42}$ The ineffective targets or benchmarks were the New Zealand Ministry of Health's 'faster cancer treatment' standards of service provision for melanoma patients, with a target of histopathological diagnosis of melanoma reported within five working days in $80 \%$ of cases, and all cases reported in 10 working days. ${ }^{15}$ In addition, two unpublished articles from Canada ${ }^{105}$ and the $\mathrm{UK}^{107}$ focusing on multiple cancers were identified, and these were the '2-week wait' benchmark in the UK (already discussed under rapid referral pathways) and the Canadian Breast Cancer Screening Network targets for diagnostic intervals: $\geq 90 \%$ of abnormal screens to be resolved within 5 weeks if no biopsy is required and $\geq 90 \%$ within 7 weeks if a tissue biopsy is required.

\section{Innovative interventions to enhanced care in cancer pre- diagnosis phase}

This review identified 17 published articles related to technological interventions for enhanced care in the prediagnosis phase of cancer. ${ }^{16} 21222937385157586265667982878991$ Ten of these articles were from the UK, ${ }^{22} 293738515762656691$ two articles were from New Zealand ${ }^{79} 82$ and one article each was from Denmark, ${ }^{89}$ Netherlands, ${ }^{21}$ Italy, ${ }^{16}$ India ${ }^{87}$ and Spain. ${ }^{58}$ These publications focused on varied cancer types in adult patient populations, with two also involving paediatric patients. The interventions had little patient input in their design, development or implementation. The focus and metrics for assessment of the effectiveness of the interventions varied across the publications. The main identified interventions were the use of teledermatology in skin cancer diagnosis. This involved the taking of images, including dermoscopy by GPs and sending them for evaluation to specialised dermatologists. ${ }^{386279} 89$ The process is embedded in an e-referral system developed in Auckland, New Zealand for suspected skin malignancy ${ }^{82}$ and included teledermatology images triaged as confirmed, likely or suspected melanoma, the use of a web-based referral tool for head and neck cancers at two different hospitals in Birmingham, West Midlands, and Wexham, Berkshire, UK. ${ }^{51}$ There was also the use 
of the Digitally Assembled Referral Toolkit for 2-week referral, accessible via a cloud-based template, which contained new referral forms native to GP clinical systems in the UK. ${ }^{29}$ Additionally, there was the use of an electronic straight-to-test pathway at a large tertiary referral hospital in England, UK to remove hospital-based triage from suspected colorectal cancer pathways; this allows GPs to book tests supported by a decision aid based on the NICE guidance, thus, eliminating the need for a standard referral form or triage process. ${ }^{65}$ Further, there was the use of electronic clinical decision support for melanoma in four general practices in the Southeast of England, UK, which involved the use of an electronicbased 7-point checklist to assess pigmented lesions, ${ }^{66}$ the use of machine learning algorithms in Newcastle, UK to classify patients referred on the 2-week wait pathway for suspected head and neck cancer into different diagnostic groups, although very broad ones: cancer and non-cancer, ${ }^{57}$ the use of nurse-led assessments to evaluate certain groups of patients suspected to have bowel cancer in England, the UK, ${ }^{22}$ and the use of varied smartphonebased skin and oral self-monitoring and screening applications, in England, $\mathrm{UK}^{91}$ and in the India, ${ }^{87}$ respectively. In addition, two unpublished articles from the UK were identified. ${ }^{106110}$ These were for a cancer decision support tool (computer-based programmes integrated into a GP's usual patient management system) in Gateshead, London, and a clinical web portal (CWP) electronic system in Manchester, England, with the fundamental part of the CWP being that local clinicians had to take personal responsibility for data input.

\section{Performance metrics to measure improvements in suspicion to diagnosis phase}

Varied performance metrics were identified by this review. The main metrics are summarised according to intervention type (online supplemental appendix 9). While performance metrics appear to be mainly interventiondependent, time from presentation in primary care to diagnosis and from referral from primary care to specialist consultation, appear to be the most consistent metrics used for evaluation. Performance metrics to measure patients' experience mainly centred on patients' satisfaction and QoL.

\section{Specific considerations for underserved populations}

Four published articles focused on issues related specifically to underserved populations, with all focused on remote/rural populations. ${ }^{18} 306088$ These publications were from the UK, ${ }^{60}$ Australia ${ }^{30} 88$ and Mexico. ${ }^{18}$ A fifth publication only used the patients' area of residence as part of their model. ${ }^{95}$ All of the publications were on multiple cancer types and adult populations, although one included a paediatric population. The specific considerations for underserved populations and the evidence regarding them included a publication from Scotland, the UK, a national audit of cancer diagnosis in Scottish and English general practices, exploring and comparing patient characteristics, diagnostic intervals and routes to diagnosis, ${ }^{60}$ the publication from New South Wales, Australia on a study that examined geographic variations in time intervals leading up to treatment for head and neck cancer, with assessment of differences based on remoteness of residence (regional/remote or metropolitan) at two tertiary referral centres ${ }^{88}$ a publication from Mexico City, Mexico on evaluation of a patient navigation programme to reduce referral time to cancer centres for underserved patients with a suspicion or diagnosis of cancer at a public general hospital, ${ }^{18}$ and a publication from Western Australia, a cluster-randomised controlled trial of a complex intervention to reduce time to diagnosis in rural patients with cancer with the aim of measuring the effect of community-based symptom awareness and general practice-based educational interventions on the time to diagnosis in rural patients presenting with breast, prostate, colorectal or lung cancer. ${ }^{30}$

\section{DISCUSSION}

This scoping review of 88 published and 16 unpublished documents from January 2017 to January 2021 summarises the evidence on current interventions focused on improving accurate and timely cancer diagnosis among symptomatic individuals. The identified articles were from varied study designs including case-control (most common), cross-sectional, before-and-after, and mixed methods studies, and randomised controlled trials. There was little evidence to suggest that patients were involved in the design, development or implementation of interventions to enhanced care in cancer prediagnosis phase.

The evidence suggests that interventions focused on improving accurate and timely cancer diagnosis among symptomatic individuals are active topics of research. The UK appears to be championing this area of research, contributing about half of all identified published literature and $83 \%$ of the identified unpublished literature. Of the specific cancer patient types, patients with lung cancer appear to be the most researched, ranking highest among the patient populations of published and unpublished literature. Of the studied interventions, rapid referral pathways and technology for supporting and streamlining the diagnosis process were the two most reported interventions. Overall, varied national and regional centralised or coordinated diagnostic services, interventions to enhance diagnostic services, multidisciplinary team approaches, patient navigation approaches, rapid referral pathways, standardised care pathways, support for PCP, target or benchmarks, technologies to support diagnosis process, and insights regarding variations between remote/rural and urban populations have been reported although there were no articles that focused specifically on Indigenous populations. Many of these intervention types could be adapted to suit different health systems and jurisdictions around the world.

The interventions mostly comprised multiple interventions/changes to the healthcare pathway. As such, the 
interventions examined varied widely across the studies. This was true even when applied to the same cancer patient populations and in the same jurisdictions/countries, including those where an intervention was part of the standard care pathway. As such, it is difficult, perhaps impossible, to identify one main approach alone that drives an intervention. Methodological approaches also varied significantly with regard to outcome assessment. A common theme among the effective centralised or coordinated diagnostic services, interventions to enhance diagnostic services, patient navigation approaches and standardised care pathways is multidisciplinary collaboration and the involvement of a nurse navigator.

The findings from this scoping review compare considerably with those of the previously summarised evidence (prior to the ongoing COVID-19 pandemic) not included in this review. ${ }^{9}$ However, while the previous evidence summary identified similar leading interventions to enhance seamless and coordinated cancer care in symptomatic individuals, intervention effectiveness was not summarised to enable comparison with the findings from this current review. As a result, assessment of the potential impact of the COVID-19 pandemic on intervention effectiveness was not possible; despite reports of decline and delays in cancer diagnosis of symptomatic individuals even in jurisdictions that use interventions that have been found to be effective from this review. ${ }^{114}{ }^{115}$ A survey by the Canadian Cancer Survivor Network showed that $54 \%$ of those surveyed (with about $75 \%$ of prediagnosis and recently diagnosed patients among them) have had their cancer care appointments cancelled, postponed or rescheduled because of COVID-19. ${ }^{116}$ Further, a modelling study in England, by Maringe and colleagues concluded that substantial increases should be expected in the number of avoidable cancer deaths as a result of diagnostic delays due to the COVID-19 pandemic. ${ }^{117}$ The conclusions of the available evidence reviews suggest that cancer screening programmes and diagnoses in symptomatic individuals, have been clearly interrupted since the onset of the COVID-19 pandemic, with delayed diagnosis and marked increases in the numbers of avoidable cancer deaths. ${ }^{18119}$

It was difficult to determine a specific intervention or a stand-alone approach to an intervention from this scoping review. It was also difficult to assess the true effectiveness of many of the interventions, especially considering the differing composite nature of the interventions, the fact that the evidence is mostly from observational studies, and the range of outcome measures used to measure effectiveness. While many of the interventions could be adapted to suit different health systems and jurisdictions, emphasis should be on the context and the strengths and limitations of the individual health system, and a clear evidence-based performance metric for appropriate evaluation of effectiveness of an intervention ought to be determined a priori. Diagnosing cancer faster and more accurately at an earlier stage is a key priority of the 20192029 Canadian Strategy for Cancer Control. ${ }^{120}$ Over the next 5 years, the Canadian Partnership Against Cancer will leverage findings from this scoping review, as one of several inputs, and partner with Canadian jurisdictions to continue to test innovative models of care that expedite cancer diagnosis, especially for Indigenous and underserved populations.

\section{Limitations and merits}

There are some limitations to this study. The literature search was developed by a knowledge synthesis librarian and peer-reviewed by an independent knowledge synthesis librarian using the PRESS checklist. We searched appropriate databases and websites for literature, and adhered to known guidelines and standards in the conduct and reporting of the review. Even so, the literature search was limited to evidence from the last 4 years and only evidence from English-language publications and organisational websites. As such, potentially eligible articles could have been missed.

The eligibility criteria for inclusion were not limited to only comparative studies. This meant that the focus of some of the included studies was not specifically on the assessment of effectiveness of an intervention and therefore, effectiveness may have been underreported for some interventions. Moreover, an intervention's effectiveness assessment was based solely on author-determined outcome, which may or may not have been an appropriate outcome for assessing effectiveness of certain interventions. As such, an intervention that appeared effective in a study may be ineffective in another study depending on the assessed outcome, with no clear reason for such a discrepancy. Furthermore, this review did not assess effectiveness of interventions across cancer patient types and jurisdictions/regions. This would have allowed assessment of any differences in intervention effectiveness by patient type and study jurisdiction. Finally, and in line with the JBI's guidance for the conduct of scoping reviews, we did not attempt to provide an assessment of the quality of the evidence and, as such, the risk of bias in randomised controlled trials and quality assessment of observational studies, including assessment for important potential biases such as selection, case ascertainment and measurement biases, and potential confounders in studies were not considered in this review; hence, the findings on effectiveness are not conclusive of the performance of the interventions.

\section{CONCLUSIONS}

The evidence suggests that interventions focused on improving accurate and timely cancer diagnosis among symptomatic individuals are active topics of research, particularly in lung cancer patient populations, and that the UK is championing this area of research. While the themes of the studied interventions are similar, the interventions differ in many ways within the same intervention group. Multidisciplinary cooperation and involvement of a nurse navigator appeared to be unique features of many of the effective interventions. 
Canadian and other jurisdictions can leverage these lessons learnt to develop and implement strategies adapted to local health system needs to improve the cancer prediagnosis phase. Future research should examine the effectiveness of the interventions identified through this review.

\section{Author affiliations}

${ }^{1}$ George and Fay Yee Centre for Healthcare Innovation, Max Rady College of Medicine, Rady Faculty of Health Sciences, University of Manitoba, Winnipeg, Manitoba, Canada

${ }^{2}$ Neil John Maclean Health Sciences Library, University of Manitoba, Winnipeg,

Manitoba, Canada

${ }^{3}$ Canadian Partnership Against Cancer (the Partnership), Toronto, Ontario, Canada ${ }^{4}$ Knowledge Translation Program, St. Michael's Hospital, Unity Health, Toronto, Ontario, Canada

${ }^{5}$ Epidemiology Division and Institute for Health Policy, Management, and Evaluation, Dalla Lana School of Public Health, University of Toronto, Toronto, Ontario, Canada ${ }^{6}$ Queen's Collaboration for Health Care Quality, Joanna Briggs Institute (JBI) Centre of Excellence at Queen's University, Kingston, Ontario, Canada

${ }^{7}$ Community Health Sciences, Max Rady College of Medicine, Rady Faculty of Health Sciences, University of Manitoba, Winnipeg, Manitoba, Canada

Contributors Conceptualisation (AP, JS, WZ, ACT); methodology (GNO, AP, JS, WZ, ACT and AMA-S); data acquisition (GNO, OLTL, VKR, LC, NA and AMA-S); formal analysis (GNO); draft manuscript (GNO and AMA-S); final manuscript revisions (GNO, OLTL, VKR, LC, NA, AP, JS, SRK, RL, WZ, ACT and AMA-S); guarantor (GNO and AMA-S). The corresponding author (the manuscript's guarantor) attests that all listed authors meet authorship criteria and that no others meeting the criteria have been omitted.

Funding This study was funded in part by the Canadian Partnership Against Cancer (the Partnership) and the Canadian Institutes of Health Research (CIHR) under the Strategy for Patient Oriented-Research (SPOR) initiative, through the SPOR Evidence Alliance. ACT is funded by a Tier 2 Canada Research Chair in Knowledge Synthesis.

Map disclaimer The inclusion of any map (including the depiction of any boundaries therein), or of any geographic or locational reference, does not imply the expression of any opinion whatsoever on the part of BMJ concerning the legal status of any country, territory, jurisdiction or area or of its authorities. Any such expression remains solely that of the relevant source and is not endorsed by BMJ. Maps are provided without any warranty of any kind, either express or implied.

\section{Competing interests None declared.}

Patient consent for publication Not applicable.

Provenance and peer review Not commissioned; externally peer reviewed.

Data availability statement All data relevant to the study are included in the article or uploaded as supplementary information.

Supplemental material This content has been supplied by the author(s). It has not been vetted by BMJ Publishing Group Limited (BMJ) and may not have been peer-reviewed. Any opinions or recommendations discussed are solely those of the author(s) and are not endorsed by BMJ. BMJ disclaims all liability and responsibility arising from any reliance placed on the content. Where the content includes any translated material, BMJ does not warrant the accuracy and reliability of the translations (including but not limited to local regulations, clinical guidelines, terminology, drug names and drug dosages), and is not responsible for any error and/or omissions arising from translation and adaptation or otherwise.

Open access This is an open access article distributed in accordance with the Creative Commons Attribution Non Commercial (CC BY-NC 4.0) license, which permits others to distribute, remix, adapt, build upon this work non-commercially, and license their derivative works on different terms, provided the original work is properly cited, appropriate credit is given, any changes made indicated, and the use is non-commercial. See: http://creativecommons.org/licenses/by-nc/4.0/.

\section{ORCID iDs}

George N Okoli http://orcid.org/0000-0002-6437-930X

Otto L T Lam http://orcid.org/0000-0001-5803-6768

Viraj K Reddy http://orcid.org/0000-0002-8526-4026

Leslie Copstein http://orcid.org/0000-0002-1295-8182
Nicole Askin http://orcid.org/0000-0001-9211-4694

Andrea C Tricco http://orcid.org/0000-0002-4114-8971

Ahmed M Abou-Setta http://orcid.org/0000-0003-0153-9916

\section{REFERENCES}

1 GBD 2015 Mortality and Causes of Death Collaborators. Global, regional, and national life expectancy, all-cause mortality, and cause-specific mortality for 249 causes of death, 1980-2015: a systematic analysis for the global burden of disease study 2015 . Lancet 2016;388:1459-544.

2 Sung H, Ferlay J, Siegel RL, et al. Global cancer statistics 2020: GLOBOCAN estimates of incidence and mortality worldwide for 36 cancers in 185 countries. CA Cancer J Clin 2021;71:209-249.

3 The Canadian Cancer Society. Cancer statistics at a glance, 2021. Available: https://bit.ly/2OjjxOO

4 Smetana K, Lacina L, Szabo P, et al. Ageing as an important risk factor for cancer. Anticancer Res 2016;36:5009-17.

5 World Health Organization. Fact sheets: cancer, 2018. Available: https://bit.ly/3c0xaL4

6 Peters MDJ, Marnie C, Tricco AC, et al. Updated methodological guidance for the conduct of scoping reviews. JBI Evid Synth 2020;18:2119-26.

7 Tricco AC, Lillie E, Zarin W, et al. PRISMA extension for scoping reviews (PRISMA-ScR): checklist and explanation. Ann Intern Med 2018; $169: 467-73$.

8 McGowan J, Sampson M, Salzwedel DM, et al. PRESS Peer Review of Electronic Search Strategies: 2015 Guideline Statement. J Clin Epidemiol 2016;75:40-6.

9 Canadian Partnership Against Cancer. Leading practices to create a seamless patient experience for the pre-diagnosis phase of care: an environmental scan. Toronto, Canada: Canadian Partnership Against Cancer, 2018.

10 Agnarsdóttir M, Päären H, Vassilaki I. The impact of standardized care pathway on reporting time for invasive melanoma - results from one pathology department in Sweden. Ups J Med Sci 2019;124:260-4.

11 Almuammar A. Primary health care factors associated with late presentation of cancer in Saudi Arabia. $J$ Radiother Pract 2020;19:71-5

12 Alonso-Abreu I, Alarcón-Fernández O, Gimeno-García AZ, et al. Early colonoscopy improves the outcome of patients with symptomatic colorectal cancer. Dis Colon Rectum 2017;60:837-44.

13 Antel K, Louw VJ, Maartens G, et al. Diagnosing lymphoma in the shadow of an epidemic: lessons learned from the diagnostic challenges posed by the dual tuberculosis and HIV epidemics. Leuk Lymphoma 2020;61:1-5.

14 Arhi CS, Burns EM, Bottle A, et al. Delays in referral from primary care worsen survival for patients with colorectal cancer: a retrospective cohort study. Br J Gen Pract 2020;70:e463-71.

15 Brian T, Adams B, Jameson M. Cutaneous melanoma: an audit of management timeliness against New Zealand guidelines. $N Z$ Med $J$ 2017;130:54-61.

16 Cazzaniga S, Castelli E, Di Landro A, et al. Mobile teledermatology for melanoma detection: assessment of the validity in the framework of a population-based skin cancer awareness campaign in northern Italy. J Am Acad Dermatol 2019;81:257-60.

17 Chapman C, Thomas C, Morling J, et al. Early clinical outcomes of a rapid colorectal cancer diagnosis pathway using faecal immunochemical testing in Nottingham. Colorectal Dis 2020;22:679-88.

18 Chavarri-Guerra Y, Soto-Perez-de-Celis E, Ramos-López W, et al Patient navigation to enhance access to care for underserved patients with a suspicion or diagnosis of cancer. Oncologist 2019;24:1195-200.

19 M C, Ic C, S S, Chng M, Coulter IC, Surash S. Impact of the updated NICE referral pathway for patients with suspected brain cancer on a neuroscience service. Br J Neurosurg 2020:1-5.

20 Christensen HM, Huniche L. Patient perspectives and experience on the diagnostic pathway of lung cancer: a qualitative study. SAGE Open Med 2020;8:2050312120918996.

21 Chung Y, van der Sande AAJ, de Roos KP, et al. Poor agreement between the automated risk assessment of a smartphone application for skin cancer detection and the rating by dermatologists. J Eur Acad Dermatol Venereol 2020;34:274-8.

22 Cock K, Evans D, Collins R. Nurse-Led service development for suspected bowel cancer. Cancer Nursing Practice 2017;16:28-31.

23 Common JL, Mariathas HH, Parsons K, et al. Reducing wait time for lung cancer diagnosis and treatment: impact of a multidisciplinary, centralized referral program. Can Assoc Radiol J 2018;69:322-7. 
24 Cotton C, Mahut C, Blyth J, et al. Using lean to improve wait time performance in diagnostic assessment for lung cancer. Healthc $Q$ 2020;22:59-63.

25 Creak A. Prospective cohort of referrals to a cancer of unknown primary clinic, including direct access from primary care. Clin Oncol 2020;32:e87-92.

26 Dahl TL, Vedsted P, Jensen $\mathrm{H}$. The effect of standardised cancer pathways on Danish cancer patients' dissatisfaction with waiting time. Dan Med J 2017;64.

27 Di Girolamo C, Walters S, Gildea C, et al. Can we assess cancer waiting time targets with cancer survival? a population-based study of individually linked data from the National cancer waiting times monitoring dataset in England, 2009-2013. PLoS One 2018;13:e0201288.

28 Drudge-Coates L, Khati V, Ballesteros R, et al. A nurse practitioner model for the assessment of suspected prostate cancer referrals is safe, cost and time efficient. Ecancermedicalscience 2019;13:994.

29 Eastham R, Duffy S, Foster C, et al. Dart: a new look at the 2 -week wait suspected cancer referral process. Br J Gen Pract 2017;67:560.

30 Emery JD, Gray V, Walter FM, et al. The improving rural cancer outcomes trial: a cluster-randomised controlled trial of a complex intervention to reduce time to diagnosis in rural cancer patients in Western Australia. Br J Cancer 2017;117:1459-69.

31 Evans J, Ziebland S, MacArtney JI, et al. Gps' understanding and practice of safety netting for potential cancer presentations: a qualitative study in primary care. Br J Gen Pract 2018;68:e505-11.

32 Evison M, Hewitt K, Lyons J, et al. Implementation and outcomes of the rapid programme: addressing the front end of the lung cancer pathway in Manchester. Clin Med 2020;20:401-5.

33 Ezer N, Navasakulpong A, Schwartzman K, et al. Impact of rapid investigation clinic on timeliness of lung cancer diagnosis and treatment. BMC Pulm Med 2017;17:178.

34 Fallon M, Adil MT, Ahmed K, et al. Impact of 'two-week wait' referral pathway on the diagnosis, treatment and survival in upper and lower gastrointestinal cancers. Postgrad Med $J$ 2019;95:470-5.

35 Gardner D, Nixon IJ. An analysis of waiting times in patients with thyroid cancer. Surg 2020;18:e51-4.

36 Hennessy M, Ryan D, Clarke S, et al. Optimal timing of CT scanning in the rapid access lung cancer clinic. Ir Med J 2020;113:121.

37 Hirst Y, Lim AWW, . Acceptability of text messages for safety netting patients with low-risk cancer symptoms: a qualitative study. $\mathrm{Br} \mathrm{J}$ Gen Pract 2018;68:e333-41.

38 Hunt WTN, Ali L, Marder H, et al. A service evaluation between 2-week wait (2WW) skin cancer referrals via teledermatology and the standard face-to-face pathway at a teaching hospital. Clin Exp Dermatol 2020;45:473-6.

39 lachina M, Jakobsen E, Fallesen AK, et al. Transfer between hospitals as a predictor of delay in diagnosis and treatment of patients with Non-Small Cell Lung Cancer - a register based cohortstudy. BMC Health Serv Res 2017;17:267.

40 Jefferson L, Atkin K, Sheridan R, et al. Non-attendance at urgent referral appointments for suspected cancer: a qualitative study to gain understanding from patients and GPs. Br J Gen Pract 2019;69:e850-9.

41 Jensen H, Tørring ML, Vedsted P. Prognostic consequences of implementing cancer patient pathways in Denmark: a comparative cohort study of symptomatic cancer patients in primary care. BMC Cancer 2017;17:627.

42 Jeyakumar HS, Wright A. Improving regional lung cancer optimal care pathway compliance through a rapid-access respiratory clinic. Intern Med J 2020;50:805-10.

43 Jiang T, Ren S, Li X, et al. The changing diagnostic pathway for lung cancer patients in Shanghai, China. Eur J Cancer 2017;84:168-72.

44 Jiang L, Gilbert J, Langley $\mathrm{H}$, et al. Is being diagnosed at a dedicated breast assessment unit associated with a reduction in the time to diagnosis for symptomatic breast cancer patients? Eur J Cancer Care 2018;27:e12864.

45 Jones JA, Catton J, Howard G, et al. Impact of straight to test pathways on time to diagnosis in oesophageal and gastric cancer. BMJ Open Qual 2018;7:e000328.

46 Joyce K, Zermanos T, Badrinath P. Factors associated with variation in emergency diagnoses of cancer at general practice level in England. J Public Health 2020:fdaa142.

47 Kassirian S, Dzioba A, Hamel S, et al. Delay in diagnosis of patients with head-and-neck cancer in Canada: impact of patient and provider delay. Curr Oncol 2020;27:e467-77.

48 Kidney E, Greenfield S, Berkman L, et al. Cancer suspicion in general practice, urgent referral, and time to diagnosis: a population-based GP survey nested within a feasibility study using information technology to flag-up patients with symptoms of colorectal cancer. BJGP Open 2017;1:bjgpopen17X101109.

49 Laerum D, Brustugun OT, Gallefoss F, et al. Reduced delays in diagnostic pathways for non-small cell lung cancer after local and national initiatives. Cancer Treat Res Commun 2020;23:100168.

50 Geraldine L, Peter B, Heather D, Largey G, Briggs P, Davies H, et al. The Victorian lung cancer service redesign project: impacts of a quality improvement collaborative on timeliness and management in lung cancer. Intern Med J 2020:08:08.

51 Lau K, Wilkinson J, Moorthy R. A web-based prediction score for head and neck cancer referrals. Clin Otolaryngol 2018;43:15:15.

52 Laudicella M, Walsh B, Burns E, et al. What is the impact of rerouting a cancer diagnosis from emergency presentation to GP referral on resource use and survival? Evidence from a populationbased study. BMC Cancer 2018;18:394.

53 McCutchan G, Smits S, Ironmonger L, et al. Evaluation of a nationa lung cancer symptom awareness campaign in Wales. $\mathrm{Br} J$ Cancer 2020;122:491-7.

54 McKevitt EC, Dingee CK, Leung S-P, et al. Reduced time to breast cancer diagnosis with coordination of radiological and clinical care. Cureus 2017;9:e1919.

55 McKevitt E, Dingee C, Warburton R, et al. Patient navigation reduces time to care for patients with breast symptoms and abnormal screening mammograms. Am J Surg 2018;215:805-11.

56 Moodley J, Cairncross L, Naiker T, et al. From symptom discovery to treatment - women's pathways to breast cancer care: a crosssectional study. BMC Cancer 2018;18:312.

57 Moor JW, Paleri V, Edwards J. Patient classification of two-week wait referrals for suspected head and neck cancer: a machine learning approach. J Laryngol Otol 2019;133:875-8.

58 Moreno-Ramírez D, Raya-Maldonado J, Morales-Conde M, et al. Increasing frequency of seborrheic keratosis diagnoses as a favorable consequence of Teledermatology-Based skin cancer screening: a cross-sectional study of 34,553 patients. Am J Clin Dermatol 2017;18:681-5.

59 Mullin MLL, Tran A, Golemiec B, et al. Improving timeliness of lung cancer diagnosis and staging investigations through implementation of standardized triage pathways. JCO Oncol Pract 2020;16:e1202-8.

60 Murchie P, Adam R, McNair E, et al. Cancer diagnosis in Scottish primary care: results from the National cancer diagnosis audit. Eur $J$ Cancer Care 2020;29:e13234.

61 Neal RD, Barham A, Bongard E, et al. Immediate chest Xray for patients at risk of lung cancer presenting in primary care: randomised controlled feasibility trial. $\mathrm{Br} J$ Cancer 2017;116:293-302.

62 Nicholson P, Macedo C, Fuller C, et al. Patient satisfaction with a new skin cancer teledermatology service. Clin Exp Dermatol 2020;45:691-8.

63 Nilbert M, Bläckberg M, Ceberg J, et al. Diagnostic pathway efficacy for urinary tract cancer: population-based outcome of standardized evaluation for macroscopic haematuria. Scand J Urol 2018;52:237-43.

64 Nixon S, Bezverbnaya K, Maganti M, et al. Evaluation of lymphadenopathy and suspected lymphoma in a lymphoma rapid diagnosis clinic. JCO Oncol Pract 2020;16:e29-36.

65 Orchard P, Arvind N, Wint A, et al. Removing hospital-based triage from suspected colorectal cancer pathways: the impact and learning from a primary care-led electronic straight-to-test pathway. BMJ Qual Saf 2021;30:11:11.

66 Pannebakker MM, Mills K, Johnson M, et al. Understanding implementation and usefulness of electronic clinical decision support (eCDS) for melanoma in English primary care: a qualitative investigation. BJGP Open 2019;3:bjgpopen18X101635.

67 Pearson C, Poirier V, Fitzgerald K, et al. Cross-Sectional study using primary care and cancer registration data to investigate patients with cancer presenting with non-specific symptoms. BMJ Open 2020;10:e033008

68 Phillips WW, Hofferberth SC, Armitage J, et al. PD01.19 lung cancer Strategist program: a novel care delivery model to improve timeliness of diagnosis and treatment. Journal of Thoracic Oncology 2019;14:S1140.

69 Piano M, Black G, Amelung D, et al. Exploring public attitudes towards the new faster diagnosis standard for cancer: a focus group study with the UK public. Br J Gen Pract 2019;69:e413-21.

70 Price S, Spencer A, Zhang X, et al. Trends in time to cancer diagnosis around the period of changing national guidance on referral of symptomatic patients: a serial cross-sectional study using UK electronic healthcare records from 2006-17. Cancer Epidemiol 2020;69:101805. 
71 Rankin NM, York S, Stone E, et al. Pathways to lung cancer diagnosis: a qualitative study of patients and general practitioners about diagnostic and pretreatment intervals. Ann Am Thorac Soc 2017:14:742-53.

72 Round T, Gildea C, Ashworth M, et al. Association between use of urgent suspected cancer referral and mortality and stage at diagnosis: a 5-year national cohort study. Br J Gen Pract 2020;70:e389-98

73 Sagar A, Mai DVC, Divya GS, et al. A colorectal straight-to-test cancer pathway with general-practitioner-guided triage improves attainment of the 28-day diagnosis target and increases outpatient clinic capacity. Colorectal Dis 2021:23:19:19.

74 Sandager M, Jensen H, Lipczak H, et al. Cancer patients' experiences with urgent referrals to cancer patient pathways. Eur $J$ Cancer Care 2019;28:e12927.

75 Sardi A, Orozco-Urdaneta M, Velez-Mejia C, et al. Overcoming barriers in the implementation of programs for breast and cervical cancers in Cali, Colombia: a pilot model. J Glob Oncol 2019;5:1-9.

76 Scott SE, Oakley R, Møller H, et al. Tracking cancer occurrence in the 5 years after referral for suspected head and neck cancer. Oral Oncol 2020;109:104955.

77 Setyowibowo H, Hunfeld JAM, Iskandarsyah A, et al. A self-help intervention for reducing time to diagnosis in Indonesian women with breast cancer symptoms. Psychooncology 2020;29:696-702.

78 Skevington SM, Long H, Gartland N. Does quality of life feedback promote seeking help for undiagnosed cancer? Qual Life Res 2020;29:1609-19.

79 Snoswell CL, Caffery LJ, Whitty JA, et al. Cost-Effectiveness of skin cancer referral and consultation using Teledermoscopy in Australia. JAMA Dermatol 2018;154:694-700.

80 Stenman E, Palmér K, Rydén S, et al. Diagnostic spectrum and time intervals in Sweden's first diagnostic center for patients with nonspecific symptoms of cancer. Acta Oncol 2019;58:296-305.

81 Stevenson-Hornby V. A rapid-access diagnostic pathway in suspected pancreatic cancer. Nursing Times 2018;114:13-11.

82 Sunderland M, Teague R, Gale K, et al. E-referrals and teledermatoscopy grading for melanoma: a successful model of care. Australas J Dermatol 2020;61:147-51.

83 Tafuri A, Ashrafi AN, Palmer S, et al. One-Stop MRI and MRI/ transrectal ultrasound fusion-guided biopsy: an expedited pathway for prostate cancer diagnosis. World J Urol 2020;38:949-56.

84 Talwar C, McClune A, Kelly D, et al. Two-Week rule: suspected head and neck cancer referrals from a general medical practice perspective. Br J Oral Maxillofac Surg 2020;58:981-5.

85 Thalanayar Muthukrishnan P, Ratnam M, Nguyen M-T, et al. PreDiagnosis multidisciplinary tumor board and time to staging in lung cancer: the case Western MetroHealth experience. Cureus 2020;12:e6595

86 Thanapal MR, Thin N, Alagaratnam S, et al. Straight-to-test colonoscopy: has it improved the detection of colorectal cancer? A 7- year review. Surgeon 2021;19:26:26.

87 Uthoff RD, Song B, Sunny S, et al. Point-Of-Care, smartphonebased, dual-modality, dual-view, oral cancer screening device with neural network classification for low-resource communities. PLOS One 2018:13:e0207493.

88 Venchiarutti RL, Clark JR, Palme CE, et al. Influence of remoteness of residence on timeliness of diagnosis and treatment of oral cavity and oropharynx cancer: a retrospective cohort study. J Med Imaging Radiat Oncol 2020;64:261-70.

89 Vestergaard T, Prasad SC, Schuster A, et al. Introducing teledermoscopy of possible skin cancers in general practice in southern Denmark. Fam Pract 2020;37:513-8.

90 Vijayakumar B, Shahidi M, Thakkar R, et al. A novel 2-week wait lung cancer pathway starting with a telephone consultation, with patient satisfaction survey results. Future Healthc $J$ 2020;7:s15.

91 Walter FM, Pannebakker MM, Barclay ME, et al. Effect of a skin self-monitoring smartphone application on time to physician consultation among patients with possible melanoma: a phase 2 randomized clinical trial. JAMA Netw Open 2020;3:e200001.

92 Whitley EM, Raich PC, Dudley DJ, et al. Relation of comorbidities and patient navigation with the time to diagnostic resolution after abnormal cancer screening. Cancer 2017:123:312-8.

93 Williams S, Davies P, Johnson B, et al. A fast track clinic improves diagnosis and treatment times for those investigated for lung cancer in Northland district health board. N Z Med J 2018;131:29-37.

94 Williams VL, Narasimhamurthy M, Rodriguez O, et al. DermatologyDriven quality improvement interventions to decrease diagnostic delays for Kaposi sarcoma in Botswana. J Glob Oncol 2019;5:1-7.

95 Fí Y, Çimen F, Ş A. Factors affecting delay in diagnosis and treatment of lung cancer. Journal of Surgery \& Medicine 2020;4:720-4.
96 Zhu J, Chen R, Davidsson S, et al. Psychological and physiological impacts of a fast-track diagnostic workup for men with suspected prostate cancer: preliminary report from a randomized clinical trial. Cancer Commun 2020;40:239-42.

97 Zienius K, Chak-Lam I, Park J, et al. Direct access CT for suspicion of brain tumour: an analysis of referral pathways in a populationbased patient group. BMC Fam Pract 2019;20:118.

98 ACE Programme. Somerset integrated lung cancer pathway. phases one and two final report final report. In: Group SCC, ed. UK: NHS, 2017: 1-12.

99 NHS England, Cancer Research UK and Macmillan Cancer Support. Improving diagnostic pathways for patients with suspected colorectal cancer. In: Accelerate CEP, ed. UK: NHS England, Cancer Research UK and Macmillan Cancer Support, 2017: 39.

100 NHS England. Improving diagnostic pathways for patients with vague symptoms. In: Accelerate CEP, ed. UK. UK: NHS England, 2017: 49.

101 NHS England, Cancer Research UK and Macmillan Cancer Support. Pharmacy training for early diagnosis of cancer. In: accelerate CEP, ed. UK: NHS England, Cancer Research UK and Macmillan Cancer Support, 2017: 27.

102 NHS England. Streamlining the lung diagnostic pathway (A87). In: trust CCCGwS, Sussex Healthcare NHS, eds. UK: NHS England, 2017: 14.

103 NHS England. Streamlining the lung diagnostic pathway (A14). In: Trust H, Mid Sussex CCGwB, Sussex University H, eds. UK: NHS England, 2017: 27.

104 NHS England, Cancer Research UK and Macmillan Cancer Support. Multidisciplinary Diagnostic Centre (MDC) based pathways for patients with non-specific but concerning symptoms. Interim report. In: Accelerate CEP, ed. UK: NHS England, Cancer Research UK and Macmillan Cancer Support, 2018.

105 New Brunswick Cancer Network. Cancer system performance 2019. New Brunswick, Canada: Government of New Brunswick, 2020: 36.

106 Gill B. Improving diagnostic pathways for patients with suspected lung cancer. In: Accelerate CEP, ed. UK: NHS England, Cancer Research UK and Macmillan Cancer Support, 2017.

107 Hamilton M, Hodgson O, Dai D. Waiting times for suspected and diagnosed cancer patients. 2017-18 annual report. In: NHS, ed. UK NHS England, 2018: 21.

108 Robinson S, Fuller E. South Tees optical referral project (STORP). A project summary. In: Accelerate CEP, ed. UK: NHS England, 2017: 14.

109 Robinson S, Fuller E. A lung health service - Doncaster pharmacy direct referral for chest X-ray. A project summary. In: Accelerate CEP, ed. UK: NHS England, Cancer Research UK and Macmillan Cancer Support, 2017: 12.

110 Robinson S, Poirier V, Watson S. Using cancer decision support tools to support the early diagnosis of cancer. In: Accelerate CEP, ed. UK: NHS, 2017: 39

111 Services AH. Cancer SCN quarterly update. July to September 2018. Canada: Alberta Health Services, 2018.

112 Stacey C, D S, M E, K S, A T. Implementing new models and standards for earlier and faster diagnosis of cancer. Paper presented at: Health and Care Innovation Expo2018; Manchester, UK 2018.

113 Inzetta SL, Musarra LL. Breast care access project. Oncology Issues 2018;33:34-45.

114 Patt D LG. M D, et al. impact of COVID-19 on cancer care: how the pandemic is delaying cancer diagnosis and treatment for American seniors. JCO Clin Cancer Inform 2020;4:1059-71.

115 Jacob L, Loosen SH, Kalder M, et al. Impact of the COVID-19 pandemic on cancer diagnoses in general and specialized practices in Germany. Cancers 2021;13:408-11.

116 Chen-See S. Disruption of cancer care in Canada during COVID-19. Lancet Oncol 2020;21:e374

117 Maringe C, Spicer J, Morris M, et al. The impact of the COVID-19 pandemic on cancer deaths due to delays in diagnosis in England, UK: a national, population-based, modelling study. Lancet Oncol 2020;21:1023-34

118 Alkatout I, Biebl M, Momenimovahed Z, et al. Has COVID-19 affected cancer screening programs? A systematic review. Front Oncol 2021:11:675038.

119 Riera R, Bagattini Ângela Maria, Pacheco RL, et al. Delays and disruptions in cancer health care due to COVID-19 pandemic: systematic review. JCO Glob Oncol 2021;7:311-23.

120 Canadian Partnership Against Cancer. Canadian strategy for cancer control (2019 to 2029): doing together what cannot be done alone. Toronto, Canada: Canadian Partnership Against Cancer 2019. 